\title{
LEARNING BASED FIDUCIAL DRIVEN REGISTRATION (LeFiR): EVALUATING LASER ABLATION CHANGES FOR NEUROLOGICAL APPLICATIONS
}

\author{
Tao Wan ${ }^{1 *}$, B.Nicolas Bloch ${ }^{2}$,Shabbar Danish ${ }^{3}$, Anant Madabhushi ${ }^{1}$ \\ ${ }^{1}$ Department of Biomedical Engineering, Case Western Reserve University, OH 44106, USA \\ ${ }^{2}$ Department of Radiology, Boston University School of Medicine, MA 02118, USA \\ ${ }^{3}$ Department of Neurosurgery, Robert Wood Johnson Medical School, NJ 08901, USA
}

\begin{abstract}
In this work, we present a novel learning based fiducial driven registration (LeFiR) scheme. We also investigate a key problem concerning the nature of landmark choices in relation to different aspects of the deformation, such as force direction, magnitude of displacement, deformation location, and native imaging artifacts of noise and intensity non-uniformity. In this work we focus on the problem of attempting to identify the optimal configuration of landmarks for recovering deformation between a target and a moving image via a thin-plate spline (TPS) based registration scheme. Additionally, we employ the LeFiR scheme to model the localized nature of deformation introduced by a new treatment modality - laser induced interstitial thermal therapy (LITT) for treating neurological disorders. Magnetic resonance guided LITT has recently emerged as a minimally invasive alternative to craniotomy for local treatment of brain diseases (such as glioblastoma multiforme (GBM), epilepsy). There is thus a need to understand (in terms of imaging features) the precise changes in the target region of interest following LITT. In order to evaluate LeFiR, we tested the scheme on a synthetic brain dataset and in two real clinical scenarios for treating GBM and epilepsy with LITT. In all cases LeFiR was found to outperform a uniform landmark based TPS registration scheme.
\end{abstract}

\section{INTRODUCTION}

Laser-induced interstitial thermal therapy (LITT), coupled with magnetic resonance imaging (MRI) guidance, has emerged as a new minimally invasive and safe approach to treat brain tumors, such as glioblastoma multiforme (GBM) [1], and more recently, to treat epileptogenic foci for epilepsy [2]. LITT allows for precisely localizing heat to a target with minimal damage to normal surrounding tissues. While LITT holds significant potential to be the modality of choice for multiple diseases (brain, prostate, breast), it is currently

\footnotetext{
* Corresponding author's e-mail: tao.wan@case.edu.

This work was made possible by grants from the National Institute of Health (R01CA136535, R01CA140772, R43EB015199, R21CA167811), National Science Foundation (IIP-1248316), and the QED award from the University City Science Center and Rutgers University.
}

only practised as an investigational procedure at a few clinical centers worldwide due to lack of data on longer term patient outcome following LITT. Consequently, there is a need to employ imaging in conjunction with LITT to better understand the precise change in the focus of treatment postLITT since the changes in imaging markers could serve as a surrogate of treatment response. Therefore, a good image registration algorithm that can spatially and accurately align pre- and post-LITT MR images is necessary and critical to quantitatively capture and evaluate subtle imaging marker changes post-LITT.

Landmark-based image registration is among the most popular methods for medical image registration. However, identifying important landmarks to perform an accurate registration remains a challenging task. Although significant contributions have been made to automatic feature-based landmark detection, such as Rechberg's method [3], these methods mainly focus on optimization of global transformation and may perform poorly on recovering local deformation. Such methods hence become inappropriate for registering pre- and post-LITT images, as a small focal region is deformed after ablation of tumor and epileptogenic foci. Alternatively, one can place the landmarks on a uniform grid to spatially align pre- and post-LITT images. However, these landmarks may not represent informative landmarks due to the uniformity and sparsity of grid.

In this work, we present a learning based fiducial driven registration (LeFiR) method (see Figure 1) to accurately align pre- and post-LITT MR images to facilitate the identification of changes in MRI markers post-therapy. The assumption is that by inducing a pre-defined deformation and attempting to recover the induced deformation allow us to learn the optimal spatial configuration of landmarks. We assume that the induced deformation in the training phase is reasonably similar to the expected deformation in a new image. Therefore, this learned landmark configuration can be employed to better recover the deformation in the new image compared to an ad hoc landmark configuration (e.g. uniform) from a registration perspective. The major contributions of this work are: (i) development of a novel LeFiR algorithm based on learning the 


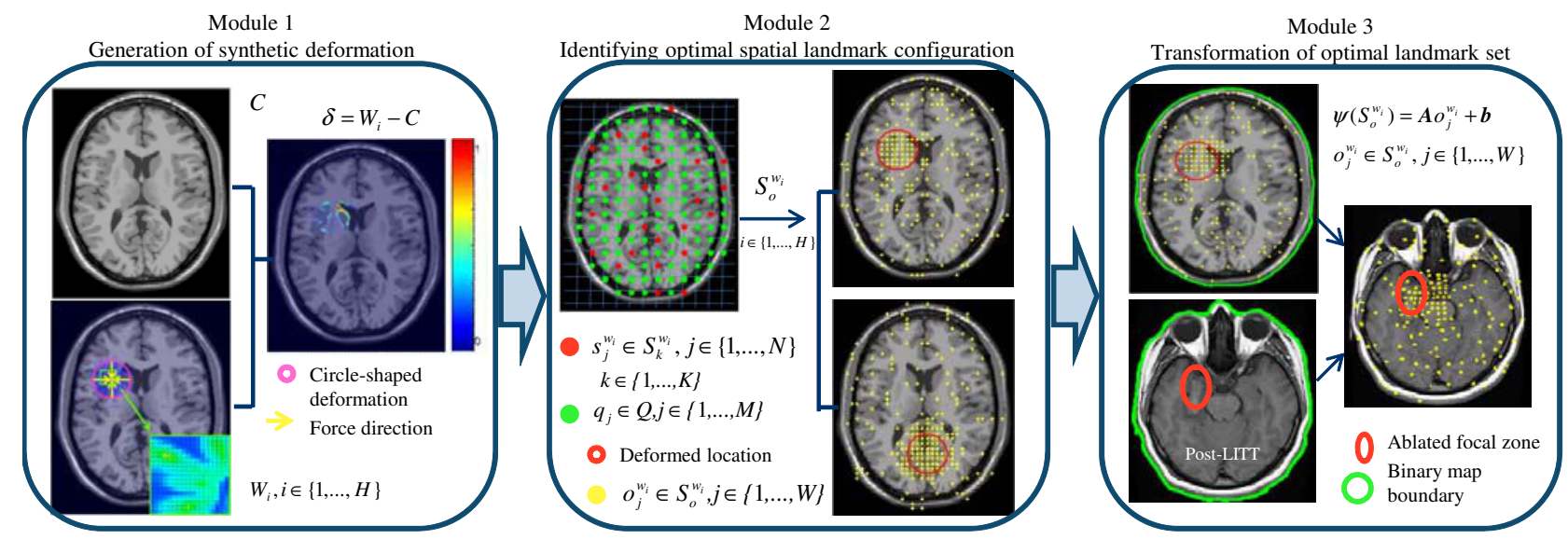

Fig. 1. The flowchart shows 3 modules of the LeFiR algorithm. Module 1 involves introducing a pre-defied deformed field on the image, the assumption being that the induced deformation will be similar to what one may expect to see in a new image. Module 2 selects important landmarks to form optimal configurations. Two examples of identified landmark sets are shown by using deformation fields $\mathcal{D}\left(f_{i}, l_{a}, m_{s}, n_{0}, u_{0}\right)$ and $\mathcal{D}\left(f_{o}, l_{b}, m_{s}, n_{5}, u_{20}\right)$, respectively. Module 3 computes a new landmark set by transforming the learned landmarks to clinical data.

optimal spatial configuration of landmarks from a registration perspective, (ii) identification of a reliable and generalizable spatial configuration of landmarks to accurately recover focal deformation induced by LITT.

\section{METHODOLOGY}

\subsection{Module 1: Generation of synthetic deformation}

We denote $\mathcal{C}=(C, f)$ as an original image, where $\mathcal{C}$ is a scene, $C$ is a grid of spatial locations $c \in C$, and $f$ is an intensity function associate with every spatial location $c \in$ $C$. A circle-shaped deformation field $\mathcal{D}$ is applied to a small region $R \subset C$ to simulate the effect of LITT on a local region, which can then be expressed as:

$$
\mathcal{D}(C)=\phi(c ; \mathcal{F}), \quad c \in R, \quad R \subset \mathcal{C}
$$

where $\phi$ is a transformation function that can be computed by considering three factors $\mathcal{F}$ : (i) two forces $f_{i}, f_{o}$, pushing the points towards the target center or outwards to the target boundary, to simulate tissue changes post-LITT, (ii) three locations $l_{a}, l_{m}, l_{b}$ representing three zones within the organ of interest which were employed to simulate different locations of disease within the brain, and (iii) three deformation magnitudes $m_{s}, m_{m}, m_{l}$ reflecting small, medium, and large deformation, in turn aimed to simulate different size and extent of treatment-related changes. Figure 1 (Module 1) shows an example of a synthetic deformation using $\mathcal{D}\left(f_{i}, l_{a}, m_{s}\right)$. A set of $H$ deformed images $\mathcal{W}_{i}, i \in\{1,2, \ldots, H\}$, was generated by moving the pre-defined deformation field $\mathcal{D}$ to various locations on the image.

\subsection{Module 2: Identifying optimal spatial landmark con- figuration}

The optimal landmark distributions $\mathcal{L}$ are learned through four experiments: (1) Experiment 1: $\mathcal{L}$ in $\mathcal{D}\left(f_{i}, f_{o}\right)$; (2)
Experiment 2: $\mathcal{L}$ in $\mathcal{D}\left(l_{a}, l_{m}, l_{b}\right)$; (3) Experiment 3: $\mathcal{L}$ in $\mathcal{D}\left(m_{s}, m_{m}, m_{l}\right)$; (4) Experiment 4: $\mathcal{L}$ in various levels of image noise $\left(n_{0}, n_{1}, n_{5}, n_{9}\right)$ and intensity non-uniformity (INU) $\left(u_{0}, u_{20}, u_{40}\right)$.

For each experiment, we first compute a landmark point base $P=\left\{p_{j}\right\}_{j=1}^{M}, p_{j} \in C$ where $C$ is a uniform grid of spatial locations on $\mathcal{C}$, and a corresponding point base $Q=$ $\left\{q_{j}\right\}_{j=1}^{M}$ on $\mathcal{W}_{i}$. Two brain images $\left\{\mathcal{C}, \mathcal{W}_{i}\right\}$ are registered via a thin-plate spline (TPS) [4] transform $\tau\left(\mathcal{C} ; \mathcal{W}_{i} ; S_{k}^{c} ; S_{k}^{w_{i}}\right)$, $k \in\{1, \ldots, K\}$, where $S_{k}^{c}=\left\{s_{j}^{c}\right\}_{j=1}^{N} \subset P$ and $S_{k}^{w_{i}}=$ $\left\{s_{j}^{w_{i}}\right\}_{j=1}^{N} \subset Q(N<M)$ are randomly chosen point sets for $\mathcal{C}$ and $\mathcal{W}_{i}$, respectively, and $k$ denotes the index of simulation. Let $v_{j}, j \in\{1,2, \ldots, M\}$, store the frequency of each point pair $\left\{p_{j}, q_{j}\right\}$ that participates in the registration. Four similarity metrics [5], including mutual information (MI), normalized cross-correlation (NCC), sum of squared intensity difference $\left(\mathrm{SSD}_{i}\right)$, and sum of square displacement difference $\left(\mathrm{SSD}_{d}\right)$, are utilized as selection criteria to compute the performance score for these selected points. After $K$ simulations, an average measure score is computed for each point pair $\left\{p_{j}, q_{j}\right\}$. Two subsets $S_{o}^{c}=\left\{o_{j}^{c}\right\}_{j=1}^{W}$ for $\mathcal{C}$, and $S_{o}^{w_{i}}=\left\{o_{j}^{w_{i}}\right\}_{j=1}^{W}$ for $\mathcal{W}_{i}$ containing $W(W<M)$ landmarks with the best values of $\frac{1}{v_{j}} \sum_{k=1}^{K} \varphi\left(\tau ; S_{k}^{c} ; S_{k}^{w_{i}}\right)$, $\varphi \in\left\{S S D_{i}, S S D_{d}, N C C, M I\right\}$, are selected to identify the optimal spatial configuration.

\subsection{Module 3: Transformation of optimal landmarks}

For a new unseen dataset comprising both the pre- and posttreatment images $\left\{\mathcal{C}_{\text {pre }}, \mathcal{C}_{\text {post }}\right\}$, a simple thresholding method can be employed to produce a binary mask $\mathcal{B}$ for each of $\left\{\mathcal{C}, \mathcal{W}_{i}, \mathcal{C}_{\text {pre }}, \mathcal{C}_{\text {post }}\right\}$. An affine transformation $\psi$ is applied to $S_{o}^{w_{i}}=\left\{o_{j}^{w_{i}}\right\}_{j=1}^{W}$ and $S_{o}^{c}=\left\{o_{j}^{c}\right\}_{j=1}^{W}$ to generate transformed landmark sets $\psi\left(S_{o}^{w_{i}}\right)$ and $\psi\left(S_{o}^{c}\right)$ as: 

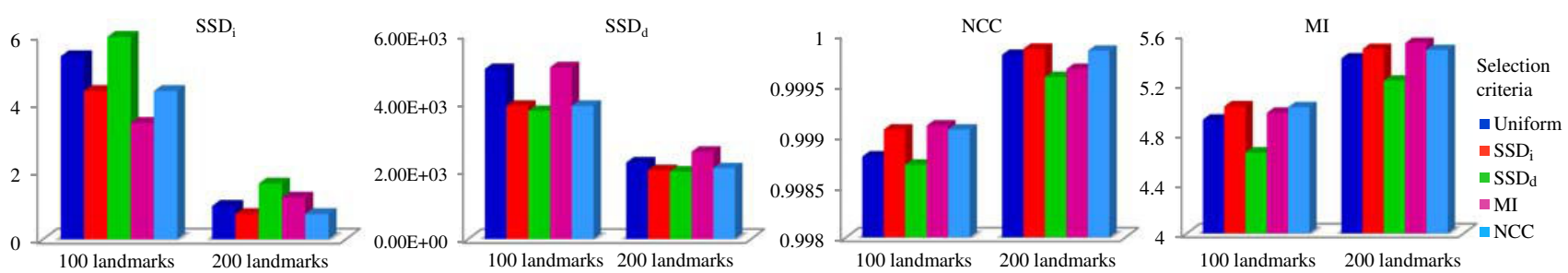

Fig. 2. Quantitative results for LeFiR on synthetic brain data using 100 and 200 selected landmarks in terms of $\mathrm{SSD}_{i}, \mathrm{SSD}_{d}, \mathrm{NCC}_{\mathrm{MI}}$. The landmark configuration using the $\mathrm{SSD}_{i}$ selection criterion achieved the best registration performance by up to $25 \%$ improvement compared to the uniformly spaced landmarks.

$$
\begin{gathered}
\psi\left(S_{o}^{w_{i}}\right)=\mathbf{A}_{\text {post }} o_{j}^{w_{i}}+\mathbf{b}_{\text {post }}, \quad o_{j}^{w_{i}} \in S_{o}^{w_{i}} \\
\psi\left(S_{o}^{c}\right)=\mathbf{A}_{\text {pre }} o_{j}^{c}+\mathbf{b}_{\text {pre }}, \quad o_{j}^{c} \in S_{o}^{c}
\end{gathered}
$$

where $\left\{\mathbf{A}_{\text {post }}, \mathbf{b}_{\text {post }}\right\}$ and $\left\{\mathbf{A}_{\text {pre }}, \mathbf{b}_{\text {pre }}\right\}$ are affine transformation matrices computed by matching two pairs of binary masks $\left\{\mathcal{B}_{\mathcal{W}_{i}}, \mathcal{B}_{\mathcal{C}_{\text {post }}}\right\}$, and $\left\{\mathcal{B}_{\mathcal{C}}, \mathcal{B}_{\mathcal{C}_{\text {pre }}}\right\}$ in the same coordinate system, respectively. Therefore, a new landmark configuration $\left\{\psi\left(S_{o}^{w_{i}}\right), \psi\left(S_{o}^{c}\right)\right\}$ is obtained by mapping the learned landmark configuration $\left\{S_{o}^{w_{i}}, S_{o}^{c}\right\}$ to the real clinical dataset. The LeFiR algorithm presented below is finally performed to spatially align two images $\left\{\mathcal{C}_{\text {pre }}, \mathcal{C}_{\text {post }}\right\}$.

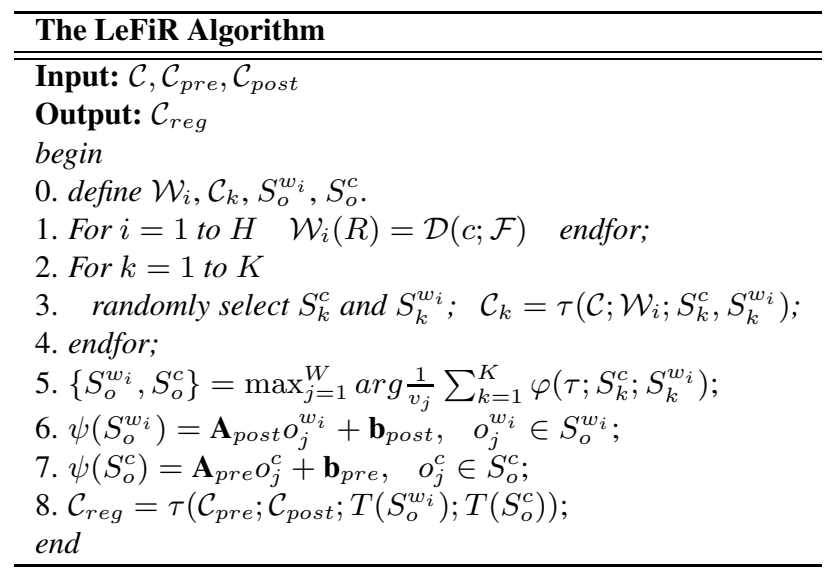

\section{EXPERIMENTAL RESULTS AND DISCUSSION}

\subsection{Dataset description}

A simulated brain database (SBD) [6] is utilized for learning optimal landmark configurations. The T1-weighted MR brain images with noise levels of $0 \%, 1 \%, 5 \%, 9 \%$, and INU levels of $0 \%, 20 \%, 40 \%$ are used in the experiments.

An FDA-cleared surgical laser ablation system (Visualase, Inc., Houston, TX) was employed for treating GBM patient and one epilepsy patient who were monitored postLITT via MRI guidance as a part of an ongoing study at the University of Medicine and Dentistry, New Jersey between 2011-2012, after initial 3-Tesla MRI. The patients were reimaged after 24 hours post LITT.

\subsection{Evaluation of learned landmark fiducials on syn- thetic brain data}

A total of $H=286$ pairs of deformed simulated brain images were generated and used in the experiments. The selected landmark configuration $\left\{S_{o}^{c}, S_{o}^{w_{i}}\right\}$ was utilized within a TPS registration scheme to register original and deformed brain MR images. The registration result was then evaluated in terms of 4 distinct quality metrics $\left(\mathrm{SSD}_{i}, \mathrm{SSD}_{d}, \mathrm{NCC}\right.$, MI). For comparison, we examined TPS registration via a uniformly spaced grid of landmark points. The same number of landmarks were used in both landmark selection strategies.

Figure 1 (Module 2) displayed two examples of the selected landmarks using two different deformation fields in terms of $\mathrm{SSD}_{i}$ as a selection criterion. The qualitative results showed that the optimally identified landmark configuration exhibited a pattern in which landmarks either within or near the deformed region were identified as being most information from a registration perspective. This trend was consistently seen across different deformation profiles and quality metrics. Figure 2 showed the performance of LeFiR evaluated by four different quality metrics $\left(\mathrm{SSD}_{i}, \mathrm{SSD}_{d}, \mathrm{NCC}\right.$, and MI) compared to the uniformly spaced landmarks. The landmark configurations identified via $\mathrm{SSD}_{i}$ and NCC suggested an up to $25 \%$ and $21 \%$ improvement in registration accuracy compared to the uniformly spaced grid points when only 100 landmarks were used.

\subsection{Co-registering pre- and post-LITT MRI in GBM}

The transformed optimal landmark set $\left\{\psi\left(S_{o}^{w_{120}}\right), \psi\left(S_{o}^{c}\right)\right\}$ was evaluated on a patient study involving GBM. Tumor was localized to one side of the brain (as shown in Figure 3(a)). The difference images between the registered and pre-LITT images were encoded in a color scale (large difference values represented in red and small values represented in blue) and overlaid on the original pre-LITT image. The identified optimal landmark configuration using the deformation field $\mathcal{D}\left(f_{o}, l_{m}, m_{s}, n_{0}, u_{0}\right)$ yielded a superior registration result with a SSD of 9.56 within the ablation site compared to a SSD of 13.75 obtained from the uniform grid. Figures 3(e),(f) suggest that the real focal deformation induced by LITT is better recovered by the learned spatial landmark configuration compared to using a uniformly spaced grid. 


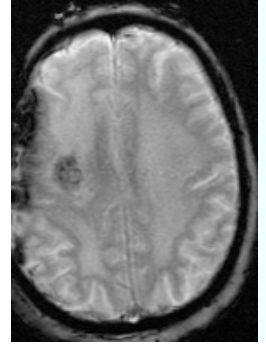

(a)

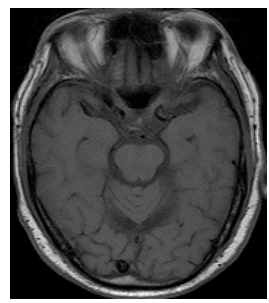

(g)

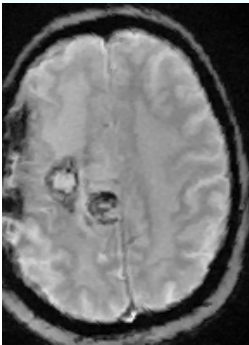

(b)

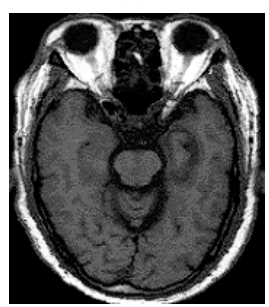

(h)

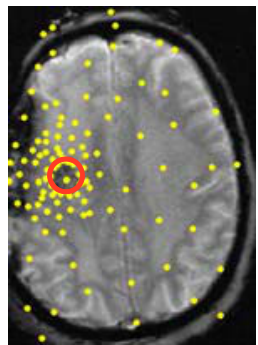

(c)

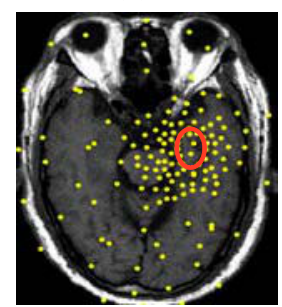

(i)

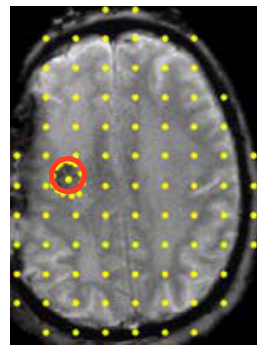

(d)

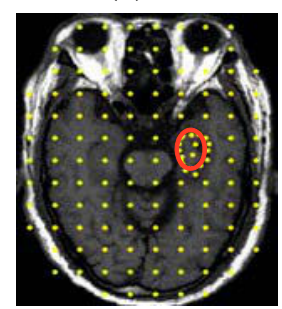

(j)

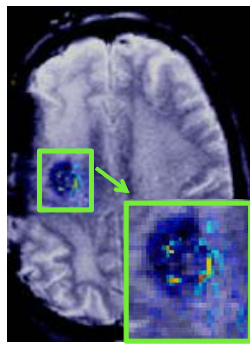

(e)

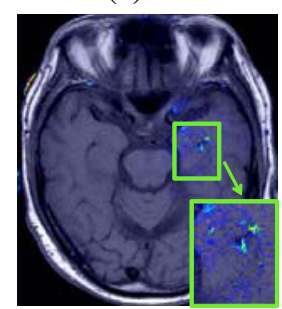

(k)

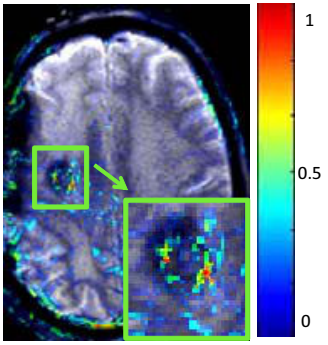

(f)

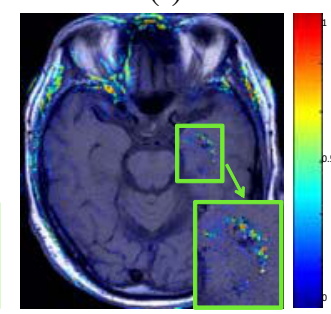

(l)

Fig. 3. Figures 3(a), (b) and (g), (h) show pre- and post-LITT brain MRI for GBM and epilepsy, respectively. Figures 3(c), (d) and (i), (j) demonstrate the landmarks (yellow points) generated by the LeFiR method and uniform grid, respectively. The red circle indicates the ablation zone. Figures 3(e),(f) and (k),(l) show the difference images between the registered and pre-LITT images using the LeFiR and uniform grid, respectively. Note that the optimal landmark configuration yielded a better registration quality compared to the uniform grid.

\subsection{Co-registering pre- and post-LITT MRI in epilepsy}

Unlike the GBM example shown in Figures 3(a)-(f), the postLITT brain MRI acquired from the epilepsy patient clearly exhibited an ablation (deformation) zone within the corresponding location of epileptogenic foci shown in the pre-LITT MRI (Figure 3(g)). An affine transformation was applied to $\left\{S_{o}^{w_{94}}\right.$, $\left.S_{o}^{c}\right\}$ to displace the points based on the location and shape of the ablation zone. The second row of Figure 3 shows the results of registering two pre- and post-LITT MR images by using the deformation field $\mathcal{D}\left(f_{o}, l_{m}, m_{m}, n_{0}, u_{0}\right)$ (Figure 3(i)) and uniform grid (Figure 3(j)) for a patient with epilepsy. The optimal landmark configuration learnt (Figure 3(i)) and uniform grid (Figure 3(j)) yielded SSD values of 6.38 and 8.55 within the ablation zone, respectively. These quantitative values were consistent with the visual examination of the difference images between the registered and pre-LITT images shown in Figures 3(k) and 3(1).

\section{CONCLUDING REMARKS}

We have presented a learning based landmark driven image registration scheme (LeFiR), in which the optimal spatial landmark configurations were learned via a supervised registration method. MRI-guided LITT provides a minimally invasive therapy for precise removal of focal abnormality (e.g. GBM, epileptogenic foci). Registration of pre- and post-LITT MRI is essential to capture and evaluate the subtle changes on the MRI following LITT. The LeFiR algorithm when tested on clinically realistic deformations was better able to capture the localized nature of deformation compared to uniform grid point placement. The findings confirmed that those configurations where the landmarks were either within or in close proximity of the deformed region in the image were more important to ensure an optimal registration result. This trend was consistently demonstrated across different deformation scenarios and similarity measures in terms of both visual and quantitative evaluations. Given that only spatial information is used to determine the optimal spatial configuration of landmarks from a registration perspective, the LeFiR method has the potential to be adopted to various clinical applications for the purposes of registering different image modalities.

\section{REFERENCES}

[1] A.Carpentier et al., "MR-guided laser-induced thermal therapy (LITT) for recurrent glioblastomas," Lasers in Surgery and Medicine, vol. 44, pp. 361-68, 2012.

[2] J.F.Tellez-Zenteno et al., "Surgical outcomes in lesional and non-lesional epilepsy: A systematic review and meta-analysis," Epilepsy Research, vol. 89, pp. 310-18, 2010.

[3] A.S.Richberg et al., "Landmark-driven parameter optimization for non-linear image registration," 2011, vol. 7962 of Proc. SPIE, pp. 1-8.

[4] A.Bartoli and A.Zisserman, "Direct estimation of non-rigid registrations," in the Proc. of BMVC, vol. 2, pp. 899-908, 2004.

[5] M.Sonka and J.M.Fitzpatick, "Handbook of medical imaging: Medical image processing and analysis," SPIE, Washington, USA, vol. 2, 2009.

[6] C.A.Cocosco et al., "Brainweb: Online interface to a 3D MRI simulated brain database," NeuroImage, vol. 5, pp. 425, 1997. 